\title{
La fugueuse chez Anne Hébert et chez Suzanne Jacob
}

ALEKSANDRA GRZYBOWSKA

UNIVERSITÉ LAVAL

Résumé. « La fugueuse chez Anne Hébert et Suzanne Jacob » a pour but de présenter les correspondances dans la construction des personnages féminins chez les deux grandes romancières québécoises. La fugueuse, figure centrale très aboutie chez Jacob, trouve peut-être, en partie, sa source dans l'œuvre d'Hébert, par exemple chez les personnages d'Élisabeth d'Aulnières, de Catherine (« Le printemps de Catherine »), surtout de Lydie (L'enfant chargé de songes), et chez les deux principaux personnages féminins du Premier Jardin. Des parallèles dans la construction du personnage de la fugueuse dans les derniers romans d'Hébert et dans les premières œuvres de Jacob sont présentés en trois volets : le portrait onomastique ou la femme-scrabble, le portrait relationnel ou la femme excessive, et le portrait spatial ou l'héroïne labyrinthique.

Mots-clés : Personnage, Fugue, Suzanne Jacob, Anne Hébert, Roman, Québec.

Anne Hébert a découvert Suzanne Jacob au début de la carrière de celle-ci, à l'occasion d'un de ses récitals parisiens. Cette rencontre de hasard a laissé son empreinte sur la personnalité et l'imaginaire de la jeune chanteuse, future poétesse et romancière. Le nom d'Anne Hébert figure parmi les auteurs dont les œuvres ont exercé sur Suzanne Jacob une grande force de séduction et transformé son imagination. De fait, la jeune romancière confessera une réelle admiration pour Anne Hébert. Les preuves en sont nombreuses. D'abord, ce sont des dédicaces émouvantes que Jacob signe dans ses romans et adresse à son amie ${ }^{1}$. Ensuite, il faut mentionner les essais de Jacob dans lesquels le nom d'Anne Hébert apparaît maintes fois. Suzanne Jacob a souligné l'importance du dialogue qui s'est établi entre elle et l'œuvre hébertienne. Ce dialogue, comme elle l'explique dans Histoires de s'entendre, « [I]'avait le mieux

1. J'ai pu lire, grâce à l'aimable collaboration de Mme Laure Miranda, auteure d'un mémoire de maîtrise intitulé Anne Hébert à l'épreuve de ses rayons : une étude quantitative de sa bibliothèque, certaines dédicaces que Suzanne Jacob a écrites pour Anne Hébert. Dans celle de La survie, par exemple, elle exprime son admiration pour Les chambres de bois et Le torrent. 
mise à l'abri de la désintégration, [...] [lui] avait fait le mieux entendre [sa] propre voix intérieure ». (Jacob, 2008 : 24) Or, certaines structures descriptives et discursives qui dominent la fiction jacobienne font écho à la pratique romanesque d'Anne Hébert. Le personnage de la fugueuse, emblématique du roman jacobien, est l'une des manifestations les plus importantes de cette correspondance entre leurs œuvres. Cette figure centrale très aboutie chez Jacob présente des affinités avec les personnages féminins de certains romans d'Hébert, par exemple Flora Fontanges du Premier Jardin, Lydie Bruneau et Camille Jouve de L'enfant chargé de songes, Héloïse, ou encore Delphine d'Est-ce que je te dérange?

Le personnage de la fugueuse constitue une variante de la femme errante. Sans entrer dans un débat définitionnel sur le concept de l'errance, je voudrais néanmoins attirer l'attention sur deux acceptions de l'errance que je développe dans l'étude du personnage de la fugueuse. Le verbe errer, comme l'explique Karin Schwergtner, « retient de sa famille historique les deux notions, erreur et voyager, en exprimant, au niveau littéraire, commettre une erreur, se tromper, et au niveau usuel, aller d'un côté et de l'autre sans but ni direction précise. » (Schwergtner, 2005 : 11) Errer couvre d'abord le plan spatio-temporel, mais, employé au sens figuré, il évoque également l'errance qui «touche l'espace de l'esprit, de la pensée, de l'inconscient». (Schwergtner, $2005:$ 12) Le personnage de la fugueuse représente une femme à la personnalité vacillante et à l'identité changeante (errance psychique et morale) qui se construit dans et à travers de multiples relations avec l'autre et par le mouvement spatial (errance physique).

Pour établir des correspondances entre certains personnages féminins de l'œuvre romanesque d'Anne Hébert et des romans de Suzanne Jacob, je présenterai trois aspects de la fugueuse ${ }^{2}$ selon le système de la description anthropomimétique propre à l'approche pragmatique du personnage littéraire ${ }^{3}$. Ainsi, $j^{\prime}$ aborderai dans le premier volet, intitulé «La femme-scrabble», la question des descriptions onomastiques qui permettent de comprendre la nature erratique de certains personnages féminins. Je me pencherai, dans la partie suivante, intitulée «La femme excessive », sur des éthopées, et plus précisément, sur le portrait interpersonnel de la fugueuse, lequel révèle son rapport à I'autre. Enfin, une réflexion sur la construction du personnage

2. Aleksandra Grzybowska. La fugueuse et ses avatars dans I'œuvre romanesque de Suzanne Jacob, Katowice, Wydawnictwo Uniwersytetu Slaskiego, 2009 : 60-135.

3. Vincent Jouve, L'effet-personnage dans le roman, Paris, Presses universitaires de France, 1992; La poétique du roman, Paris, SEDES, 1999; Pierre Glaudes et Yves Reuter, Le personnage, Paris, Presses universitaires de France, $1998: 91$. 
dans l'espace urbain présentera la dimension spatiotemporelle de la fugueuse, complétant ainsi son portrait global (« L'héroïne labyrinthique »).

\section{La femme-scrabble}

Figure protéiforme par excellence, la fugueuse est dotée de plusieurs prénoms, noms ou pseudonymes qui peuvent paraître énigmatiques. On peut l'appeler femmepuzzle, femme-scrabble ou encore femme-kaléidoscope. Ces désignations expriment à l'unisson des traits sine qua non de la fugacité : l'être désintégré et en devenir ainsi que la variété des masques portés changent en fonction des lieux, des personnes et des situations. De nombreux romanciers et critiques littéraires ont mis en relief I'importance du choix du nom, qui constitue une étape déterminante dans la création du personnage. Roland Barthes, notamment, explique que

le Nom propre est lui aussi un signe, et non bien entendu, un simple indice qui désignerait, sans signifier, comme le veut la conception courante, de Peirce à Russell. Comme signe, le Nom propre s'offre à une exploration, à un déchiffrement : il reste à la fois un « milieu » (au sens biologique du terme), dans lequel il faut se plonger, baignant indéfiniment dans toutes les rêveries qu'il porte, et un objet précieux, comprimé, embaumé, qu'il faut ouvrir comme une fleur. Autrement dit, si le Nom (on appellera ainsi, désormais, le nom propre) est un signe, c'est un signe volumineux, un signe toujours gros d'une épaisseur touffue de sens (Barthes, $1972: 125$ ).

Anne Hébert comme Suzanne Jacob dotent leurs héroïnes d'un éventail significatif de noms, de prénoms et de surnoms qui annoncent d'intéressants projets diégétiques. Prenons l'exemple de l'évolution onomastique de I'héroïne du Premier Jardin. Elle reflète son parcours existentiel et biographique qui, malgré les sinuosités de la quête identitaire, reste très balisé. Les trois noms du personnage, comme trois cartes d'identité, correspondent aux grandes périodes de la vie de l'héroïne. Pierrette Paul est le premier nom de la jeune fille, « dépossédée de sa propre mère, depuis la nuit des temps »(Le Premier Jardin, 1988 : 100), qui reste malgré tout sa grande héritière. Pierrette, abandonnée à I'hospice Saint-Louis à Québec, tient de sa mère, comme un mal incurable, le réflexe involontaire de fuir. La mère de Pierrette, cette grande absente qui demeure obsessionnellement présente, incarne par excellence la fugueuse qui a enfermé la génération postérieure des femmes dans le cercle infranchissable des fuites (songeons également à Maud, fille de Flora, qui est fugueuse de naissance). Marie Éventurel est le deuxième nom propre de l'adolescente miraculeusement sauvée de l'incendie de I'hospice Saint-Louis. Si I'on réfléchit à la sémantique de ce nouveau nom propre, il est possible d'y découvrir une annonce assez précise 
de l'avenir de l'héroïne comme de sa mission à accomplir dans la société québécoise. Le prénom de Marie renvoie à la Sainte Vierge, dont de nombreux auteurs québécois ont fait un symbole d'obéissance, de soumission et de passivité. Telle devait rester Marie Éventurel, soumise aux règles de la haute bourgeoisie à laquelle appartenaient les Éventurel. Le patronyme de ces derniers annonce le modèle que la jeune héroïne voudrait incarner « éventurellement » : la féminité libérée et épanouie (Marie-Ève, la synthèse de la sainte et de la tentatrice en une seule personne), le naturel et l'ouverture à I'aventure, à l'inconnu. Flora Fontanges est le troisième nom propre de I'héroïne, qui évoque, comme l'explique Lori Saint-Martin, « les fleurs, [...] les fontaines, les anges, tout ce qui rappelle, de près ou de loin, le premier jardin ». (Saint-Martin, 1999 : 197) En développant cette piste d'interprétation, on peut aller plus loin pour constater que le nom propre de l'héroïne signifie le retour au premier jardin, c'està-dire, le retour au paradis, ou jardin d'Éden, lieu où elle puisse devenir ce qu'elle désire être ${ }^{4}$. En effet, ce nom devient « le seul et l'unique qui la désignera entre tous et lui permettra toutes les métamorphoses nécessaires à sa vie. » $\left(P J^{5}: 65\right)$ Femme libre et libérée, Flora Fontanges choisit la profession d'actrice, qui lui procure « la jubilation incomparable de passer à travers le noyau éclaté de son cœur, en flammèches ardentes. » ( $P J: 171)$ Elle se cherche constamment, tantôt dans ses rôles théâtraux, pour « essayer une autre peau que la sienne » $(P J: 63)$, pour oublier son drame et vivre ceux de Phèdre, de Célimène, d'Ophélie, de Desdémone ${ }^{6}$, tantôt dans I'histoire de Marie Rollet, des filles du Roi et des orphelines mortes pendant I'incendie de I'hospice Saint-Louis.

En ce qui concerne les héroïnes de Suzanne Jacob, elles sont dotées d'une pléthore de noms propres et communs dont l'analyse permet de saisir, dans une certaine mesure, les dédales intérieurs de fugueuses. Leurs diverses appellations témoignent du parcours psychogéographique et biographique? ${ }^{7}$. Le nom propre chez Jacob est souvent dépositaire de projets diégétiques. Laura Laur, revendiquant deux fois plutôt qu'une le prénom de la muse de Pétrarque, inspire et trouble la vie de ses deux

4. « [J]e m'appelle Marie Éventurel, je ferai tout ce que vous me direz de faire et je serai telle que vous me voulez, comme il vous plaira, jusqu'à ce que mon désir d'être une autre me reprenne, me pousse et me tire loin de vous et de la ville, au-delà des mers, là où je serai actrice, dans les vieux pays. » (Le Premier Jardin, 1988 : 65)

5. PJ pour Le Premier Jardin, 1988.

6. « Je m'appelle Phèdre, Célimène, Ophélie, Desdémone. Je retombe sur mes pieds après chaque représentation. Je salue bien bas. Puis je vaque à mes petites affaires, comme tout le monde. » (PJ : 171)

7. «[I]l y a diverses architectures de Galatée, suivant les villes et les circonstances. » (Jacob, $1986: 235$ ) 
frères et de ses deux amants, qui expriment dans les quatre parties du roman, chacun son tour, leur attachement à cette femme fascinante. La double initiale L.L. évoque, comme l'explique Lori Saint-Martin, le pronom féminin (elle-elle), qui « fait sonner le féminin à l'état presque pur. » (Saint-Martin, $1996: 253)^{8}$ Obnubilé par la beauté remarquable et dérangeante de Laura, Gilles ne voit en elle que son corps, dont émanent une frénésie et une fureur de vivre. Il appelle immanquablement par son prénom cette femme fantasmagorique devenue réelle qui lui inspire les folies de la deuxième jeunesse, la passion péremptoire qui exige tout. Laura se montre plus énigmatique et insaisissable pour Pascal, son deuxième amant. Quand il lui demande comment elle s'appelle, elle lui répond que cela dépend des jours, qu'elle peut être Madeleine, Jeanne ou Laura'. De son côté, le prénom de l'héroïne éponyme de La passion selon Galatée rappelle celui de la statue mythique sculptée par Pygmalion. La Galatée de Jacob comme celle de Pygmalion attirent I'attention. Leur présence n'est jamais anodine; elle bouleverse la vie des autres et le cours des événements. Galatée est plus souvent appelée Gala, pseudonyme fréquent mais réservé uniquement aux intimes. Ses amoureux et ses amoureuses le déclinent à six cas possibles, telle la rosa latine : «Gala, Gala, Galae, Galae, Gala, Galam. » (Jacob, 1986 : 159) Le mode de vie de Gala porte bien des signes d'insouciance et de légèreté, comme si elle était toujours à la fête, ce qui renvoie à l'étymologie du mot (d'origine italienne, espagnole ou portugaise), qui signifie magnificence, réjouissance.

La fugueuse jacobienne, comme celle d'Anne Hébert, connaît la règle d'or du mimétisme et excelle à se transformer comme un caméléon en fonction des personnes et des circonstances. Gala confesse :

Je pouvais fabriquer quatre Gala différentes le même soir selon les goûts et les désirs de chacun. Je me soumettais avec bonheur aux règles du mimétisme qui harmonisaient les styles de mes édifices. Tout pouvait s'écrouler, il n'y avait pas de conséquences à ces fabrications, si ce n'est pour l'un ou l'autre qui s'était attaché ou habitué trop rapidement à telle ou telle invention. J'avais la certitude que le mimétisme constituait le fond de tout. Souvent, je me prenais sur le fait d'accomplir des gestes ou des pensées de ma sœur Titi, de mon père ou d'Augustine, de Cyrille ou de Baldwin. (Jacob, $1986: 88-89)$

8. Nous pensons surtout à I'interprétation sémantique de Laura Laur proposée par Lori Saint-Martin. La critique ajoute encore que le nom de Laura Laur « pourrait se lire comme une mise en texte de I'androgynie : forme féminine (en «a ») et masculine d'un même nom. » (Saint-Martin, $1996: 255$ )

9. Pascal, photographe, ne se lasse pas de la prendre en photo pour tenter d'éterniser ce qui est insaisissable et fugace. Les photos monothématiques ne présentent qu'un seul objet d'adoration, Laura, ses yeux, ses mains, son corps. 
L'aveu de Flora Fontanges complète celui de Gala :

Éclater en dix, cent, mille fragments vivaces; être dix, cent, mille personnes nouvelles et vivaces. Aller de l'une à l'autre, non pas légèrement comme on change de robe, mais habiter profondément un autre être avec ce que cela suppose de connaissance, de compassion, d'enracinement, d'effort d'adaptation et de redoutable mystère étranger. ( $P J: 64)$

En somme, les héroïnes d'Hébert et de Jacob excellent à entrer dans la peau de I'autre et elles y vivent mieux que dans la leur. Elles s'y identifient spontanément, et cette identification constitue par la suite un excellent moyen de se fuir. L'évolution onomastique des noms propres d'Anne Hébert et de Suzanne Jacob, leurs significations révèlent un penchant manifeste pour la désintégration intérieure et l'identité vacillante qui est en train de se chercher et de se définir.

\section{La femme excessive}

Certaines héroïnes de Jacob et d'Hébert sont des femmes hors du commun. Inspirées et inspirantes, elles font penser aux muses ou encore aux médiums, un peu à I'image des personnages ou des égéries des surréalistes français ${ }^{10}$. Elles savent lire dans le tréfonds de l'âme et amener les êtres à donner libre cours à leurs fantasmes et à leurs pulsions les plus profondes. La fugueuse est, comme le cocktail Molotov, une créature enflammée et explosive qui marque d'un sceau celui ou celle qu'elle côtoie. C'est un être de la démesure et de l'étrangeté dont les sentiments et les attitudes sortent du cadre. La frénésie des désirs, l'ivresse des sentiments, la volonté de toute-puissance ou l'hybris tout court, comme diraient les Grecs, constituent le trait caractéristique du portrait psychologique de la fugueuse. À l'origine de la révolte orgueilleuse et souvent spontanée contre la logique du monde, contre les conventions, contre les lois morales, un penchant naturel pour l'hybris motive les décisions et les démarches de ces personnages féminins d'Hébert et de Jacob. Ce trait de personnalité se construit tout particulièrement dans et par leurs rapports interpersonnels et s'intensifie dans un contexte bien particulier. On peut en effet noter des ressemblances dans les modalités de la représentation de l'hybris de la fugueuse dans les derniers romans d'Anne Hébert et dans les premières œuvres de Suzanne Jacob : la fugueuse survient de nulle part (tel le Grand-dieu-des-routes de Germaine Guèvremont), soudainement et de manière très remarquée, et impose sa présence à l'autre, lequel se laisse envoûter par son charme; elle surgit dans un univers sédentaire qui 
baigne dans l'immobilisme et qui exclut la possibilité du moindre changement, dont elle bouleverse les habitudes et croyances.

On découvre, dans Héloïse (1980) d'Anne Hébert, I'histoire de Bernard, jeune homme qui semble le plus heureux du monde parce qu'il aime Christine et est aimé d'elle. Il est résolu, sans l'ombre d'un doute, à passer toute sa vie avec son amoureuse, lorsqu'un jour la voix envoûtante d'une belle inconnue secoue sa torpeur amoureuse :

Une voix de femme émerge peu à peu du fracas du métro. Acidulée, ironique, persifleuse, elle se rapproche de plus en plus. Elle chante. On distingue bientôt les paroles. Bernard éprouve la sensation aiguë qu'on s'adresse à lui. [...] Cette voix étrange qu'il n'arrive pas à localiser (il y a tant de monde dans le wagon) atteint Bernard au plus profond de luimême. En guise de défense il se croit obligé d'écrire, à la pointe feutre, sur un panneau publicitaire : « Bernard aime Christine. Christine aime Bernard. » La voix ricane dans I'ombre. (Héloïse, 1980 : 19-20)

Lydie Bruneau, dans L'enfant chargé de songes (1992), constitue la femme excessive par excellence. Cette adolescente de dix-sept ans habite passagèrement chez les Ouellet, des gens discrets et ordinaires qui incarnent l'esprit monotone et tranquille de la petite campagne de Duchesnay. La première apparition publique de la jeune fille dévoile sa personnalité rebelle et ses penchants anarchistes. Lydie, en se manifestant d'une manière très spectaculaire dans la rue principale du village, bouleverse l'ordinaire de ses habitants : «Eux tous qui étaient captifs d'une vie austère et monotone, voici qu'ils vivaient une aventure étonnante qui les clouait sur place de surprise et de contentement. » (L'enfant chargé de songes, 1992 : 43) Vêtue d'un short modeste, Lydie arrive en plein midi sur un cheval qui « allait au pas dans la rue de l'église, les jambes nues de la fille qui le montaient à cru étaient fort écartées à cause de la taille énorme du cheval de labour » $\left(E C S^{11}: 42\right)$.

On trouve le même goût pour la provocation chez les personnages féminins des trois premiers romans de Suzanne Jacob. Laura Laur, I'héroïne éponyme du roman paru en 1983, joue un rôle important dans I'histoire personnelle de quatre hommes, soit deux frères et deux amants. Elle apparaît à l'improviste dans la vie des hommes, qu'elle rend entièrement dépendants d'elle. Laura choque, dérange, renverse le cours des événements et l'ordre présupposé des choses. Gilles, par exemple, son premier amant, apprécie grandement l'édifice de sa sécurité : 
Je suis un homme d'un certain âge. J'ai une femme, une famille, une maison. Je suis un homme qui a réussi. J'ai cette chance de n'avoir jamais été victime du destin. Le destin pour moi est un programme ou une programmation. Ma chance dans la vie tient à ce qu'il n'y a pas d'erreur dans mon programme. Je suis un homme respecté. J'ai des économies en prévision de ma retraite. (Jacob, $1999: 56)$

La jeune femme, une erreur dans la vie programmée et sécurisée à l'excès de I'homme respecté, prend du plaisir à démolir avec une certaine lenteur l'édifice du succès professionnel et «la mécanique sereine » (Jacob, 1999:45) de son confort conjugal et familial. En déséquilibrant la situation personnelle de Gilles, Laura le contamine et lui communique ce qu'on pourrait appeler un certain courage de vivre, d'un certain point de vue. Elle est l'incarnation même de la frénésie mêlée à l'ivresse de la vie. Il en va de même avec Flore Cocon, I'héroïne du premier roman de Jacob (1978). Récalcitrante et impulsive comme Lydie, Flore Cocon bouleverse totalement les habitudes quotidiennes de ses amis et des nouveaux venus. Elle se glisse imperceptiblement dans le quotidien des hommes (Pierre, son amoureux, Marc, son amant, et bien d'autres adorateurs : Laurent, Maxime...) ainsi que des femmes (Josette Rowan, Louanne). La liste exhaustive en serait longue, et les cas, trop nombreux à exposer ici.

Les expressions de l'hybris sont diverses (la tenue provocante de Lydie, la voix charismatique d'Héloïse, les comportements amoraux de Flore Cocon ou de Laura Laur) et influent d'une manière déterminante sur la nature des liens interpersonnels de la fugueuse. Ce qui apparaît certain, c'est qu'il n'y aura jamais d'accord ni d'harmonie durables entre la fugueuse, cette nomade moderne, et le personnage sédentaire, profondément attaché aux valeurs conventionnelles de sa famille ou de sa communauté. Cependant, l'excès agit comme un puissant aphrodisiaque, décuplant l'attrait de la différence et l'intensité de l'échange entre les opposés. La démesure trouve son expression la plus adéquate dans le processus de la séduction.

Belles et inaccessibles, les héroïnes hébertiennes suscitent des fantasmes érotiques et des pulsions indomptables chez leurs amoureux. Elles deviennent l'objet des rêveries et des désirs inassouvis. Annabelle M. Rea ${ }^{12}$ a justement remarqué que I'œuvre romanesque d'Anne Hébert se déploie sous le signe de Dionysos. Celui que Nietzsche opposait au raisonnable Apollon représente des «moments de folie, certes un peu délirants, excessifs à coup sûr, mais aussi ludiques que joyeux, jusque dans l'excès justement, bref, ces instants d'aimable transgression qu'une vie "libérée" doit dé- 
dier à I'hédonisme, au plaisir, à la satisfaction des passions érotiques même les plus secrètes ». (Ferry, 2008 : 379) Personnage hébertien peut-être le plus dionysiaque, Lydie Bruneau, associée à la passion des chevaux ${ }^{13}$, éprouve des sentiments intenses et des sensations à l'état brut où se mêlent toujours les passions les plus obscures, la sexualité dévoilée, le sadisme, le mal. Elle révèle, par sa tenue et ses comportements troublants ${ }^{14}$, par ses paroles habiles, des zones insoupçonnables dans la psyché et dans le corps des jeunes hommes. Lydie devient la muse de Julien en lui inspirant ses premiers vers et des phrases d'amour, tandis qu'elle se comporte comme une « allumeuse » en réveillant chez Alexis les pulsions les plus bestiales. Apparue dans la vie de Julien lorsqu'il séjourne à Paris, Camille Jouve se présente elle-même à lui comme une «passante, sans passé et sans avenir. » (ECS : 148) «En face d'elle qui est vivante et rieuse il se sent comme un convalescent, à peine sorti d'une étrange maladie sans nom précis ». (ECS : 20) Camille éveille en lui la pulsion de vie, et ces mêmes désirs charnels qui brûlaient son corps à côté « des jambes interminables » (ECS : 22) de Lydie, dont Camille apparaît comme un double.

Les démarches troublantes, les paroles séduisantes des personnages dionysiaques chez Anne Hébert mènent certains de leurs amoureux à la mort ${ }^{15}$. Héloïse, le personnage vampirique, incarne à la fois Éros et Thanatos, deux pulsions inséparablement liées, qui ne se rencontrent qu'une fois dans l'intimité :

Bernard tombe aux pieds d'Héloïse. Il enfouit sa tête dans les jupes de la jeune femme. Retrouve l'odeur prenante des grèves; varech, goémon, vase profonde qui fume et se déchaîne. Il enserre les jambes d'Héloïse jusqu'à la faire tomber par terre. Ils roulent tous les deux sur le tapis. Un bref cri de douleur.

Est-ce moi qui crie, pense Bernard, pendant que la volupté le broie et l'emmène jusqu'aux portes de la mort. $\left(\begin{array}{lll}H^{16} & : 100\end{array}\right)$

\footnotetext{
13. Voir à ce propos l'essai de France Nazair Garant intitulé Ėve et le cheval de grève : contribution à l'étude de l'imaginaire d'Anne Hébert, Québec, Centre de recherche en littérature québécoise, Université Laval, 1988.

14. «Alexis ne pouvait se rendre compte si elle était vraiment belle ou tout simplement étrange. Mais il se souvenait de ses longues cuisses écartées lorsqu'elle était sur le cheval. » (ECS : 44)

15. L'amour démesuré qu'Elisabeth Tassy a pour George Nelson devient mortifère (Kamouraska).

16. $H$ pour Hélö̈se, 1980.
} 
Lydie Bruneau apprend à la petite Hélène la désobéissance envers sa mère et la maîtrise de soi au prix des épreuves qu'elle doit subir. La course à bicyclette en est la première ${ }^{17}$ et la course dans les rapides en est la dernière :

Elle n'est plus qu'obéissance à la maîtresse du canot qui avironne à droite ou à gauche, rétablissant l'équilibre de minute en minute. Déjà l'écume, en longs crachats blancs, file dans le courant de plus en plus agité, tandis que le fracas devient étourdissant. [...] Le canot rouge danse et craque au gré des flots. Lydie et Hélène entrent toutes les deux dans l'intimité de la mort. (ECS : 104)

En ce qui concerne les comportements provocants et sentiments enflammés des héroïnes jacobiennes, ils n'engendrent pas la mort mais la transgression. Les fugueuses jacobiennes sont des femmes désobéissantes parce qu'elles ne se retrouvent ni dans le cadre familial, ni dans le cadre social qui leur est imposé. Elles enfreignent tout ce qui représente la norme, le dogme ${ }^{18}$, en poussant leurs adorateurs à faire la même chose. Provocante et rebelle, Flore Cocon séduit le mari de sa meilleure amie, lequel, sans hésiter, se lance dans l'aventure amoureuse et sensuelle. Dans Rouge, mère et fils, Delphine Laurier entretient une relation amoureuse avec Lenny, I'ami de son fils. Consciente de son charme et de l'influence qu'elle exerce sur les hommes, la fugueuse jacobienne s'avère plus forte et experte en jeu de séduction que I'héroïne d'Anne Hébert. Prête à séduire ou à être séduite ${ }^{19}$, I'héroïne jacobienne se plaît délibérément à draguer les hommes, à éveiller leur pulsions pour y succomber :

\begin{abstract}
Alors, Flore fait des choses magnifiques avec des hommes éblouis qui s'abîment en elle voluptueusement. Flore ne se fait pas prier pour assumer l'hypnose qu'elle exerce sur les hommes. Chacun d'eux a son histoire. Chacun d'eux dose l'affection, mesure la tendresse qu'il faut montrer pour se coucher, avec prudence. Parcimonieusement. Et quand ils retombent lourdement parmi les draps, Flore garde les yeux ouverts et les regarde dormir. Elle veut continuer à croire qu'elle est humaine et que ces amants, de passage entre ses cuisses, sont attendrissants. (Jacob, 1978b : 46)
\end{abstract}

17. «Elle est là, debout devant elle, inexplicablement triomphante dans sa robe sale et déchirée, les genoux ensanglantés, une grosse bosse sur le front. - C'est en revenant du lac que je suis tombée sur les cailloux. La bicyclette de Lydie est toute tordue mais Lydie dit que ça ne fait rien. L'important, c'est que j'aie pu pédaler toute seule jusqu'au lac. Lydie dit que c'est très bien. » (ECS : 79)

18. «La délinquance est un genre d'aérodynamisme [...]. Les avions et les fusées sont des objets délinquants [...] Ils défient la loi de la pesanteur, ils lui désobéissent! » (Jacob, 1999 : 164)

19. «Ça arrive. N'importe où. Vous croisez quelqu'un. Ou vous vous assoyez à la table voisine de la sienne. Une personne. Un étranger. Un autre. Ça arrive. Au-delà des formules, du savoir-vivre et des politesses, au-delà des demi-mots et des grossièretés, vous échangez un regard qui dit : "Je suis à vous. Faites de moi ce que vous voulez pour l'instant. Dans une heure ou deux ou dans dix ans, je verrai à me reprendre s'il le faut." » (Jacob, 1978b : 42) 
La fugueuse chez Jacob enflamme et maîtrise totalement les sentiments aussi bien des hommes que des femmes. Suzanne Jacob va plus loin dans la représentation de la séduction au féminin ${ }^{20}$ qu'Anne Hébert a évoquée en décrivant l'amitié amoureuse entre Lydie et Hélène dans L'enfant chargé de songes.

Ces étrangères, dont l'origine demeure imprécise, sont dotées d'une incroyable force centripète qui fascine et hypnotise. Excessives, elles séduisent en comblant le manque ou le vide de leurs amoureux ou amoureuses attachés à un milieu exempt d'excentricité. Les fugueuses fusionnent avec le monde, qu'elles bouleversent, séduisent les hommes comme les femmes, ce qui a pour conséquence qu'ils et elles s'ouvrent à une nouvelle dimension de la vie, avant de disparaître d'une manière aussi soudaine qu'elles sont apparues.

L’héroïne labyrinthique

Les fuites perpétuelles, les passages d'un lieu à l'autre, le désir de partir, le besoin d'aller voir ailleurs, bref le mouvement constant est intégralement inscrit dans l'être de la fugueuse. Ce personnage est appelé à survoler les territoires comme à vagabonder dans un lieu clos. Il reflète la condition du héros western qui ne connaît pas les frontières ${ }^{21}$ comme celle du héros labyrinthique qui erre dans des zones hostiles. Un deuxième type de fugueuse se trouve aussi exploité par les deux romancières. Les œuvres romanesques d'Anne Hébert et de Suzanne Jacob offrent un éventail fort riche d'architectures labyrinthiques parmi lesquelles la ville occupe une place privilégiée. Les toponymes et les références topographiques dans les romans d'Anne Hébert permettent de situer l'action soit à Paris (Les chambres de bois, Héloïse, L'enfant chargé de songes, Est-ce que je te dérange?, Un habit de lumière) soit au Québec (Kamouraska, Le Premier Jardin). L'espace urbain est doté, comme l'explique Daniel Marcheix, de «valeurs dysphoriques au premier rang desquelles figurent l'inauthentique et l'opacité. » (Marcheix, 2005 : 340) Le discrédit qui frappe la ville découle de son inaccessibilité, qui évide toute forme de présence. Le personnage hébertien vit une relation conflictuelle avec son environnement spatial, qui engendre des émotions négatives comme la frustration, la crainte, l'incertitude (c'est le cas par exemple de Flora Fontanges, d'Élisabeth d'Aulnières et de Delphine dans Est-ce que je te dérange?). Moins antagonistes sont les rapports des héroïnes jacobiennes, qui vivent une sorte de symbiose avec l'espace urbain. Montréal et Québec, lieux de passage,

20. Pour en savoir plus, voir Aleksandra Grzybowska, $2009: 226-247$

21. Aleksandra Grzybowska, 2009 : 281-285. 
constituent des agglomérations anonymes qui procurent, à l'opposé de l'espace privé saturé de souvenirs traumatisants, un sentiment de liberté, d'autonomie et d'indépendance 22 . Flore Cocon, Laura Laur comme Galatée rencontrent leurs amants dans des hôtels luxueux ou minables. Elles participent à des spectacles de toutes sortes, au théâtre, au restaurant, au cimetière ou dans la chambre de leurs amis. Les hôtels, les rues, les bars, les parcs et tant d'autres lieux publics sont des témoins muets des jeunes femmes, à la recherche de «leur apparition dans le monde. » (Carrier, $1992: 84)$

Les fugueuses hébertiennes et jacobiennes enfermées intra muros de la ville labyrinthique deviennent des femmes errantes ${ }^{23}$. L'errance liée inextricablement à l'éthique du nomadisme se transforme en itinérance, c'est-à-dire en parcours déconcertant, apparemment discontinu mais doté de valeurs positives. Conformément à la dialectique du labyrinthe, les fugueuses, en arpentant les villes ou en rôdant dans les ruelles, trouvent un moyen de mener une exploration, voire une quête, un moyen d'atteindre le centre mythique, celui de la vérité sur soi (je pense surtout à Flore Cocon et à La passion selon Galatée). Parmi de très nombreuses itinérances dans les romans de Jacob, la plus spectaculaire demeure celle de Marie Cholet, I'une des héroïnes de L'obéissance. À bout de forces et accablée par le procès pour infanticide (qu'elle vient de gagner), la jeune avocate erre avec son amie Julie dans le réseau de boulevards, de rues et d'impasses montréalais ${ }^{24}$. L'exploration de l'espace extérieur contribue indirectement à celle de l'espace intérieur par le réveil des expériences douloureuses d'un lointain passé. L'inconscient de l'avocate à succès, soigneusement calmée et rassurée par sa réussite professionnelle et personnelle, se manifeste d'une manière ahurissante dans le quartier chinois où se clôt sa promenade. Marie Cholet s'est exercée toute sa vie à obéir à ce qu'elle ne comprenait pas. Obéir à ses parents monstrueux qui les maltraitaient, elle et ses frères. Obéir à son mari volage. Obéir

22. Les romans de Suzanne Jacob se rapprochent de l'esthétique du roman urbain. À propos du roman de la ville, voir Jaap Lintvelt, «L'espace identitaire de la ville de Québec dans le roman québécois depuis $1960 \gg$ dans Lucie K. Morisset, Luc Noppen et Denis Saint-Jacques (dir.). Ville imaginaire, ville identitaire : échos de Québec, Québec, Nota bene, 1999 ; Pierre Nepveu et Gilles Marcotte, Montréal imaginaire, Québec, Fides, 1992.

23. «La femme errante, un peu clocharde, un peu folle, hante l'imagination féminine. Elle est si loin de la féminité socialement imposée qu'elle fascine en même temps qu'elle rebute. » (Saint-Martin, 1997 : 229)

24. Jacob consacre un chapitre de dix-neuf pages dans L'obéissance (183-202) à la relation d'une précision extrême de leur déambulation. Un itinéraire topographique se dessine au cœur du labyrinthe montréalais : le parc Lafontaine, la rue Rachel, la rue Saint-Denis, la terrasse de la Brûlerie, le carré Saint-Louis, la rue Notre-Dame, le palais de justice, la rue Saint-Laurent avec l'édifice de La Presse et, finalement, le quartier chinois. 
à l'éthique professionnelle et à une fausse image de sa personne qui se voulait forte et indépendante. Bref, obéir à cette aspiration qui la condamnait au succès professionnel, à bâtir une sorte de tour d'ivoire pour faire taire ses blessures d'autrefois. Son inconscient explose là où l'impératif d'obéir est aboli. Marie Cholet éprouve un malaise indicible et se trouve sur le point de s'évanouir dans le quartier chinois, où l'obéissance est impossible parce qu'elle ne comprend pas les signes : « Oh ! j'adore traverser le quartier chinois! Je ne comprends rien, ni la langue, ni les affiches, ni les objets : je ne sais pas à quoi servent certains objets dans les vitrines, et ça me plaît. Je ne voudrais pas que quelqu'un m'explique à quoi ça sert. » (Jacob, 1991 : 201)

Il est possible de trouver une séquence semblable dans l'itinérance de Flora Fontanges dans Le Premier Jardin, ce qui est relativement rare parce que la plupart des romans hébertiens proposent « une réactualisation spatialisée du désastre de l'individualisation. » (Marcheix, 2005 : 352) Flora parcourt sa ville natale, le labyrinthe par excellence, avec Raphaël, I'ami de sa fille, qui l'aide à identifier certains lieux et personnages historiques du Vieux-Québec. L'itinérance géographique devient itinérance psychique, morale et mémorielle ${ }^{25}$. Sur certains points de la territorialité urbaine se greffent des territoires intérieurs qui enferment les secrets et les non-dits de I'héroïne. Poussée par un désir d'« aller jusqu'au fond de sa mémoire » $(P J: 166)$ et de «[d]ébusquer tous les fantômes » $(P J: 134)$, Flora se résout à se rendre « jusqu'au bout de cette côte qui n'en finit pas » $(P J: 166)$, cette côte de la Couronne où se dressait jadis I'hospice Saint-Louis. Le lieu de la tragédie personnelle et collective revisité, le drame verbalisé et narrativisé permettent au personnage d'accueillir le manque, de convertir le refoulé en appropriation cathartique des territoires intimes et urbains.

Les descriptions métonymiques de l'espace dans Le Premier Jardin et dans L'obéissance prouvent encore une fois la désintégration de l'être des fugueuses. Mal à l'aise avec elle-même, traquée par ses secrets ou souvenirs douloureux, la fugueuse agit conformément à son instinct : elle fuit; fuir pour oublier, pour être le plus loin de l'espace qui fait mal. Mais l'analyse de la femme labyrinthique ou errante, le deuxième type de fugueuse, permet de découvrir que ces haltes obligées dans un espace hostile du labyrinthe s'avèrent salvatrices. Toute fuite est une quête. Oser arpenter le labyrinthe de l'espace douloureux comme celui de ses zones interdites signifie voyager au bout de soi-même. 
Au terme de ma relecture paradigmatique de personnages féminins dans I'œuvre romanesque de deux grandes écrivaines québécoises, après avoir établi parallèles et analogies entre les personnages et considéré ce qui caractérise leur présence, j'espère avoir montré comment Anne Hébert et Suzanne Jacob contribuent à définir le type de la fugueuse. Qui dit fugueuse dit désintégration, démesure, désobéissance et itinérance. La fugueuse est synonyme de femme libre et libérée, mais aussi cassecou. Cette héroïne aspire à la liberté généralement comprise (personnelle, familiale, sociale), et c'est pourquoi elle brave le convenu et l'interdit à son corps défendant, bafoue les frontières du possible pour se donner la chance de découvrir une nouvelle dimension de sa présence au monde - ce qui a le même effet sur ceux et celles qu'elle séduit. Rebelle et excentrique, la fugueuse désobéit aux rôles sociaux, déjoue les promesses parentales, trahit les ententes amicales. Elle paye souvent cher, par l'exclusion, son inadaptation ou son étrangeté ainsi que ses actes anarchistes. Elle est reléguée en marge de la société, par choix ou par suite de son comportement.

Anne Hébert et Suzanne Jacob appartiennent à deux générations d'écrivains québécois. De même, leurs œuvres romanesques représentent deux esthétiques différentes. Les trois portraits qu'on vient de lire témoignent de nombreuses correspondances mais aussi de différences qui se dessinent entre leurs personnages féminins. On parlerait en effet de correspondances ou de rencontres, plutôt que de filiation ou d'influence à sens unique ou réciproque, entre ces deux œuvres et ces deux auteures. Anne Hébert propose notamment dans ses derniers romans des personnages féminins qui portent en germe la révolte, la transgression. Ces personnages annoncent, en frayant la voie à une fugacité mieux circonscrite, plus explicite, mais aussi moins meurtrière, les héroïnes libres et libérées de Suzanne Jacob. Dans ce sens, on peut considérer Anne Hébert comme une prophétesse dont l'œuvre romanesque annonce le renouveau, le changement dans la représentation littéraire de la femme et de sa condition sociale. Les protagonistes des derniers romans d'Hébert et des premiers romans de Jacob, parus dans les années 1980, reflètent un système axiologique qui prend de l'ampleur après la Révolution tranquille, et qui comprend notamment l'ébranlement du schéma familial traditionnel, l'affirmation du féminisme et une poussée nouvelle de l'individualisme. En effet, Lydie Bruneau, Flora Fontanges, Flore Cocon, Laura Laur et quelques autres aspirent à une autonomie totale en refusant la famille, le patriarcat et l'identification aux rôles préétablis (la fille à marier, la reine du foyer, la mère dévouée, la vierge et la putain). Loin de ne chercher qu'à fuir les 
modèles préexistants, la fugueuse aspire à trouver son véritable centre, son identité propre, fût-ce dans le mouvement perpétuel. 


\section{Bibliographie}

BARTHES, Roland (1972), Le degré zéro de l'écriture, suivi de Nouveaux essais critiques, Paris, Seuil.

BISHOP, Neil (1997), "Guerres, errances et exils dans l'œuvre d'Anne Hébert », dans Madeleine Ducrocq-Poirier [et al.], Anne Hébert, parcours d'une œuvre. Actes du colloque de la Sorbonne, Montréal, l'Hexagone : 163-174.

CARRIER, Anne (1992), « Qui est Suzanne Jacob ? », Québec français, n 85 : 82.

FERRY, Luc (2008), La sagesse des mythes. Tome 2. Apprendre à vivre, Paris, J'ai lu.

GLAUDES, Pierre et Yves REUTER (1998), Le personnage, Paris, Presses universitaires de France.

GRZYBOWSKA, Aleksandra (2009), La fugueuse et ses avatars dans l'œuvre romanesque de Suzanne Jacob, Katowice (Pologne), Wydawnictwo Uniwersytetu Slaskiego.

GRZYBOWSKA, Aleksandra (2009), "Le nouveau nomadisme dans l'imaginaire de Suzanne Jacob », dans Krzysztof Jarosz, Zuzanna Szatanik et Joanna Warmuzinska-Rogoz (dir.), De la fondation de Québec au Canada d'aujourd'hui (1608-2008), Katowice (Pologne), Para : 277-287.

HÉBERT, Anne (1980), Héloïse, Paris, Seuil.

HÉBERT, Anne (1988), Le premier Jardin, Paris, Seuil.

HÉBERT, Anne (1992), L'enfant chargé de songes, Paris, Seuil.

HÉBERT, Anne (1998), Est-ce que je te dérange?, Paris, Seuil

JACOB, Suzanne (1978a), La survie, Montréal, Le Biocreux.

JACOB, Suzanne (1978b), Flore Cocon, Montréal, Parti Pris.

JACOB, Suzanne (1986), La passion selon Galatée, Paris, Seuil.

JACOB, Suzanne (1991), L'obéissance, Paris, Seuil.

JACOB, Suzanne (1999), Laura Laur, Montréal, Boréal.

JACOB, Suzanne (2001), Rouge, mère et fils, Paris, Seuil

JACOB, Suzanne (2008), Histoires de s'entendre, Montréal, Boréal.

JOUVE Vincent (1992), L'effet-personnage dans le roman, Paris, Presses universitaires de France.

JOUVE Vincent (1999), La poétique du roman, Paris, SEDES.

LINTVELT, Jaap (1999), « L'espace identitaire de la ville de Québec dans le roman québécois depuis 1960 », dans Lucie K. Morisset, Luc Noppen et Denis Saint-Jacques (dir.), Ville imaginaire, ville identitaire : échos de Québec, Québec, Nota bene.

MARCHEIX, Daniel (2005), Le mal d'origine. Temps et identité dans l'œuvre romanesque d'Anne Hébert. Québec, L'instant même.

NAZAIR GARANT, France (1988), Ève et le cheval de grève : contribution à l'étude de l'imaginaire d'Anne Hébert, Québec, CRELIQ.

NEPVEU, Pierre et Gilles MARCOTTE (1992), Montréal imaginaire, Montréal, Fides.

REA, Annabelle M. (2000), "La femme dionysiaque chez Anne Hébert », Les Cahiers Anne Hébert, $\mathrm{n}^{\circ} 2: 95-111$.

SAINT-MARTIN, Lori (1996), "Suzanne Jacob, à l'ombre des jeunes filles en fuite », Voix et images, vol. $31, \mathrm{n}^{\circ} 2$ (62) : 250-257. 
SAINT-MARTIN, Lori (1997), Contre-voix. Essais de critique au féminin, Québec, Nuit blanche.

SAINT-MARTIN, Lori (1999), Le nom de la mère. Mères, filles et écritures dans la littérature au féminin, Québec, Nota bene.

SCHWERDTNER, Karin (2005), La femme errante, Ottawa, Legas. 